\title{
On Cosmic Epochand Linear Size/Luminosity Evolution of Compact Steep Spectrum Sources
}

\author{
Ezeugo Jeremiah Chukwuemerie \\ Department of Physics and Industrial Physics, Nnamdi Azikiwe University, Awka, Nigeria
}

\section{Email address:}

emailchuksemerie@yahoo.com

\section{To cite this article:}

Ezeugo Jeremiah Chukwuemerie. On Cosmic Epochand Linear Size/Luminosity Evolution of Compact Steep Spectrum Sources. American Journal of Astronomy and Astrophysics. Vol. 9, No. 1, 2021, pp. 8-12. doi: 10.11648/j.ajaa.20210901.12

Received: March 4, 2021; Accepted: March 16, 2021; Published: April 7, 2021

\begin{abstract}
We have used analytical methods in this paper to obtain a mathematical relation that describes relationship between the linear size of compact steep spectrum (CSS) sources and their redshift. Result shows that the source linear size has an inverse power-law dependence on the redshift. Moreover, for the purpose of obtaining an empirical relation that shows relationship between the liner size and the redshift, we carry out simple linear regression analyses on the observed linear sizes of the CSS sources in our sample against their respective observed redshifts. Results of the analyses indicate that the linear sizes of the quasars have direct power law relationship with their respective redshifts; while the converse is the case for their galaxy counterparts. Their correlation coefficients are marginal. In comparison with the obtained theoretical relation, we notice that for the CSS quasars, the linear size-redshift data show an inverse correlation. This is comparable with the theoretical relation. So, it suggests that the dynamical evolution of the source linear sizes may have some cosmological effects on it. However, the converse is the case for the CSS galaxies - the correlation is direct. The possible explanation for this difference is that quasars are observed at higher redshifts than their galaxy counterparts. Hence, the cosmological effects are expected to be more pronounced on the quasars. Furthermore, we use analytical methods again to obtain a theoretical relation that shows relationship between luminosity and redshift. The relation indicates that luminosity of a radio source has an inverse power-law relationship with redshift. This suggestively implies that the intrinsic luminosity of a radio source may be modified by cosmological evolution. Moreover, for the purpose of obtaining an empirical relation for comparison with the theory, we carry out linear regression analysis of observed luminosities against observed redshifts of the CSS quasars and galaxies in our sample. Results show that luminosities have excellent direct power-law relationship with redshifts. However, this is in contradiction to the obtained theory which shows inverse relationship between the two parameters. This excellent direct correlation has been attributable by some authors to strong luminosity selection effects in which samples with high luminosities are found at high redshifts. Therefore, if the selection effects are taken care of, we may be able to see the comparability of the theory with the empirical relation. Hence, we conclude that source radiated power may have some cosmological implications just like we saw in the size/redshift relation.
\end{abstract}

Keywords: Radio Sources, Redshift, Steep Spectrum, Compact, Evolution, Cosmology, Luminosity

\section{Introduction}

The building blocks of the Universe are the galaxies. In terms of their luminosities, galaxies can be classified into normal galaxies and active galaxies. Active galaxies are those galaxies that radiate in excess of $10^{36} W[1-5]$. Unlike the normal galaxy whose radiation comes from the constituent stars, the active galaxy radiate copious amount of radiation from its three major components, namely, central core (believed to harbor a suppermassive black hole), two-sided jets emanating from the core, and two-sided lobes fed by the jets [1-5].

Compact steep spectrum sources (CSSs), on the other hand, belong to a class of active galaxies known as extragalactic radio sources (EGRSs) that radiate more in the radio wavelengths [6]. The major difference between the CSSs and the normal EGRSs (or extended radio sources) is their smallness but yet powerful in radiation [6-8]. They constitute a remarkable class of radio sources accounting for a substantial fraction of the extra-galactic sources selected, especially, at high radio frequencies where the source counts are usually dominated by flat spectrum (spectral index, 
$\alpha<0.5, S_{v} \propto v^{-\alpha}$; where $S_{v}$ is flux density). They are not just cores that show steep spectra, rather they are full-fledged radio galaxies and quasars complete with jets and lobes, but on small scale $[6,8]$. They have been shown to contain special characteristics that make them be considered as a separate class of objects in addition to lobe- and coredominated Active Galactic Nuclei (AGNs). They are usually found at high redshifts (generally, they tend to have redshift distribution of $z \leq 4$ ), and are among high luminosity sources.

The CSS sources are a mixture of radio galaxies and radio loud quasars with radio power, $P_{5 G H z}>10^{25.5} \mathrm{WHz}^{-1}$ and show double, triple and core-jet morphologies on the radio maps. Their projected linear sizes range from less than $1 \mathrm{kpc}$ to $20 \mathrm{kpc}$ assuming Hubble's constant, $H_{0}=75 \mathrm{Kms}^{-1} \mathrm{Mpc}^{-1}$, and deceleration parameter, $q=0$, [6]. They have steep high frequency spectrum of spectral index, $\alpha<0.5, S_{v} \propto v^{-\alpha}$. Sometimes, the spectrum remains straight up to $100 \mathrm{GHz}$, proving that no dominant flat spectrum core exists. Generally, they have their radio spectral turnovers at frequencies, sometimes $>5 \mathrm{GHz}$; and have very low polarization at both radio and optical bands, usually less than one percent $[6,7,9]$. Their proportion is high; about 15 $-30 \%$, depending on the selection frequency, among distant $(z>0.2)$ radio sources of high power $[6,7,9]$.

\section{Models of Formation and Evolution of Compact Steep Spectrum Sources}

The nature of CSSs has been discussed for many years. Immediately after their discovery, it was suggested that they might be very young radio sources; that is, the progenitors of the extended doubles [6-11]. However, it has been proposed that CSSs are, instead, old 'frustrated sources which have been kept small by a dense confining medium [6-12]. Moreover, it has been suggested that CSSs might be 'smothered' sources in which a large deposition of gas had recently confined an existing radio-loud AGN to a small volume; though this does not have any footing for want of data [6-12]. Other models include: Relativistic Beaming and Orientation Effects [13, 14]; and Hybrid Model - in which Youth, Frustration scenarios and Relativistic Beaming \& Orientation Effects are combined.

Furthermore, from simulation and statistical approach, it has been concluded that if CSSs were randomly oriented in space, only a minority $(\leq 30 \%)$ of them were larger sources of the same intrinsic radio luminosity foreshortened by projection effects [7]. This simply implies that hybrid model could be of great importance in the proper explanation of the phenomenal occurrences in the CSSs.

In this paper, we use analytical methods to obtain theoretical expressions that may show a relationship between the linear size of a radio source and its redshift, and between luminosity and redshift. After which, we find empirical relations between observed linear sizes of the CSS radio sources and their redshifts; as well as, between the observed radio luminosities and observed redshifts. These CSS sources are from [6] and they are made up of 31 CSS quasars and 28 CSS galaxies, totaling 59 sources in our sample.

\section{Cosmological Evolution of Radio Source Size}

The synchrotron aged spectrum model for CSS sources can be expressed as $[6,9]$

$$
\mathcal{T}_{\text {syn }}=1610 \frac{\mathcal{B}^{\frac{1}{2}}}{\mathcal{B}^{2}+\mathcal{B}_{C M B}^{2}}\left((1+z) v_{b r}\right)^{-\frac{1}{2}}
$$

where $\mathcal{B}$ is magnetic field intensity, $\mathcal{B}_{C M B}=3.25(1+z)^{2}$ in $\mu G$ is the magnetic field equivalent to the microwave background, $\mathcal{T}_{\text {syn }}$ in Myr is elapsed time since the source formation, $v_{b r}$ in $\mathrm{GHz}$ is the breaking frequency. Substituting for $\mathcal{B}_{C M B}$, we obtain

$$
\mathcal{T}_{\text {syn }}=1610 \frac{\mathcal{B}^{\frac{1}{2}}}{\mathcal{B}^{2}+10.56(1+z)^{4}\left((1+z) v_{b r}\right)^{\frac{1}{2}}}
$$

Simplifying, we have

$$
\mathcal{T}_{\text {syn }}=\frac{1610 \mathcal{B}^{\frac{1}{2}}}{(1+z)^{\frac{9}{2}\left[\mathcal{B}^{2}(1+z)^{-4}+10.56\right] v_{b r}^{\frac{1}{2}}}}
$$

Since $(1+z)^{\frac{9}{2}} \gg(1+z)^{-4}$, we ignore the smaller term to obtain

$$
\mathcal{T}_{\text {syn }} \approx \frac{1610 \mathcal{B}^{\frac{1}{2}}}{10.56 v_{b r}^{\frac{1}{2}}(1+z)^{\frac{9}{2}}}
$$

Moreover, kinematic age, $\mathcal{T}$, of a radio source can be expressed simply as a function of the source linear size, $\mathcal{D}_{0}$, as [14]

$$
\mathcal{T}=\int_{\mathcal{D}_{m}}^{\mathcal{D}_{0}} \frac{d \mathcal{D}_{0}}{\mathcal{V}_{\ell}}
$$

where $\mathcal{D}_{m}$ is the lower limit of the linear size, $\mathcal{V}_{\ell}$ is lobe velocity. Assuming radiation age has similar value with the kinematic age, we combine the last two equations to obtain

$$
\mathcal{D}_{0} \approx \frac{1610 B^{\frac{1}{2}} v_{\ell}}{10.56 v_{b r}^{\frac{1}{2}}(1+z)^{\frac{9}{2}}}
$$

Hence, we have

$$
\mathcal{D}_{0} \sim(1+z)^{-4.5}
$$

which can be referred to as theoretical relation. It can be interpreted to mean that the linear size of an EGRS possibly may be affected by cosmological evolution.

Moreover, for the purpose of obtaining an empirical relation for comparison with the foregoing, we carry out simple linear regression analysis on the observable source linear sizes against their respective observed redshifts (Figures 1 and 2). Figure 1 is for the CSS quasars while Figure 2 is for the CSS galaxies. Results of the regression 
show,

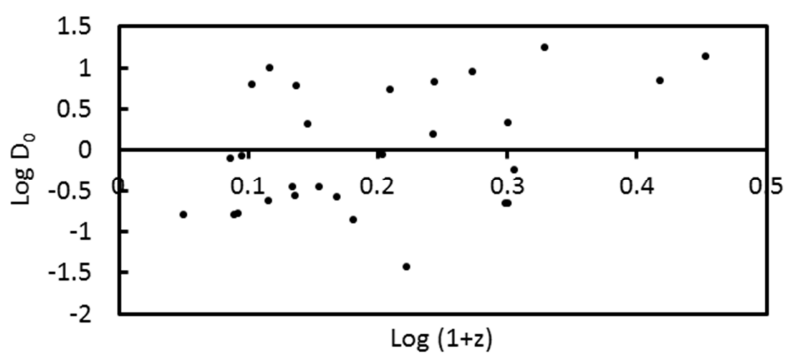

Figure 1. The scatter plot of linear size against redshift for the CSS galaxies.

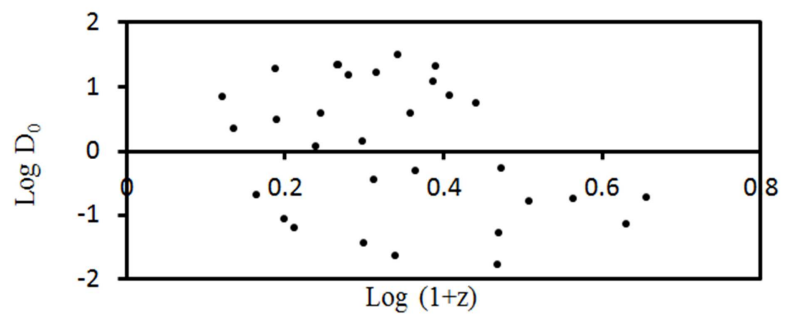

Figure 2. The scatter plot of linear size against redshift for the CSS quasars.

$$
\log \mathcal{D}_{0}=-2.49 \log (1+z)+0.89
$$

and

$$
\log \mathcal{D}_{0}=2.92 \log (1+z)-0.58
$$

for quasars and galaxies respectively. Their correlation coefficients are given respectively as 0.33 and 0.40 . These show marginal correlation. Rewriting equations (8) and (9) yields respectively

$$
\mathcal{D}_{0} \sim(1+z)^{-2.5}
$$

and

$$
\mathcal{D}_{0} \sim(1+z)^{2.9}
$$

These last two relations can be referred to as empirical relations. In comparison with the theoretical relation (equation (7)), we notice that for the CSS quasars, $\mathcal{D}_{0}-z$ data show an inverse correlation. This is in consonance with the theoretical relation. So, this suggests that there may be some effects caused by cosmological evolution on the source linear sizes.

However, the converse is the case for the CSS galaxies the correlation is direct. The possible explanation for this difference is that quasars are observed at higher redshifts than their galaxy counterparts. Hence, the cosmological effects are expected to be more visible on the quasars.

\section{Cosmological Evolution of Luminosity}

Furthermore, assuming lobe confinement by ram-pressure balance with the ambient density, we have [7, 15],

$$
\mathcal{P}_{\ell} \approx \eta m_{h} v_{l}^{2}
$$

where $\mathcal{P}_{\ell}$ is lobe internal pressure, $\eta$ is particle number density of the source ambient medium, $m_{h}$ is hydrogen mass.

It can be shown that jet kinetic power, $\mathcal{P}_{c j}$, can be expressed according to the following relation [15]:

$$
\mathcal{P}_{c j} \approx \eta m_{h} c v_{j}^{2} \Gamma \mathcal{D}_{0}^{2}
$$

where $\Gamma$ is jet opening solid angle, $c$ is light speed, and other parameters have their usual meanings. The last equation can be recast to become,

$$
\mathcal{D}_{0} \approx\left(\frac{\mathcal{P}_{c j}}{\eta m_{h} c v_{j}^{2} \Gamma}\right)^{\frac{1}{2}}
$$

By definition,

$$
\mathcal{P}_{c j}=\frac{m_{j} a \mathcal{D}_{0}}{t}
$$

where $m_{j}$ is mass of jet and $a$ is acceleration. Also, accretion rate

$$
\dot{\mathcal{M}}=\frac{\mathcal{M}_{m}}{t}
$$

$\mathcal{M}_{m}$ is the mass of accreted matter for a period of the source dynamical age, $t$. If we assume core-jet power, $\mathcal{P}_{c j}$, to be a function of accretion rate, $\dot{\mathcal{M}}$; and for simplicity $\mathcal{M}_{j} \approx \mathcal{M}_{m}(1-e)$, (where $e$ is conversion efficiency or the kinetic power into radiation, [5]), we may have

$$
\mathcal{P}_{c j}=\dot{\mathcal{M}}(1-e) \frac{\mathcal{D}_{0}^{2}}{t^{2}}
$$

where $a$ has been substituted with $\frac{\mathcal{D}_{0}}{t^{2}}$. Therefore equation (14) becomes

$$
\eta \approx\left(\frac{\dot{\mathcal{M}}(1-e)}{m_{h^{c} v_{j}^{2} t^{2} \Gamma}}\right)^{\frac{1}{2}}
$$

But it can be shown that

$$
\dot{\mathcal{M}}=\frac{\mathcal{P}_{b o l}}{e c^{2}}
$$

$\mathcal{P}_{\text {bol }}$ is bolometric luminosity. Therefore, substituting for $\dot{\mathcal{M}}$ equation (18) becomes

$$
\eta \approx\left(\frac{\mathcal{P}_{b o l}}{c^{3} e m_{h} \mathcal{D}_{0}^{2} \Gamma}\right)^{\frac{1}{2}}
$$

We have replaced $v_{j}^{2} t^{2}$ with $\mathcal{D}_{0}^{2}$. or making the linear size, $\mathcal{D}_{0}$, subject, we get

$$
\mathcal{D}_{0} \approx\left(\frac{\mathcal{P}_{b o l}}{c^{3} e m_{h} \Gamma \eta}\right)^{\frac{1}{2}}
$$

where $e \leq 1$. The last equation describes the dynamical evolution of CSS sources. With $\mathcal{P}_{b o l}$ as the subject, we obtain

$$
\mathcal{P}_{b o l} \approx c^{3} e m_{h} \Gamma \eta \mathcal{D}_{0}^{2}
$$

Hence, we have

$$
\mathcal{P}_{\text {bol }} \sim \mathcal{D}_{0}^{2}
$$


Combining with equation (7), we obtain

$$
\mathcal{P}_{b o l} \sim(1+z)^{-9}
$$

The last equation is a theoretical relation. It plausibly indicates that the luminosity (or generally, radiated power) of a radio source falls off as $(1+z)^{-9}$. Hence, showing that luminosity of a radio source may suggestively be modified by cosmological evolution.

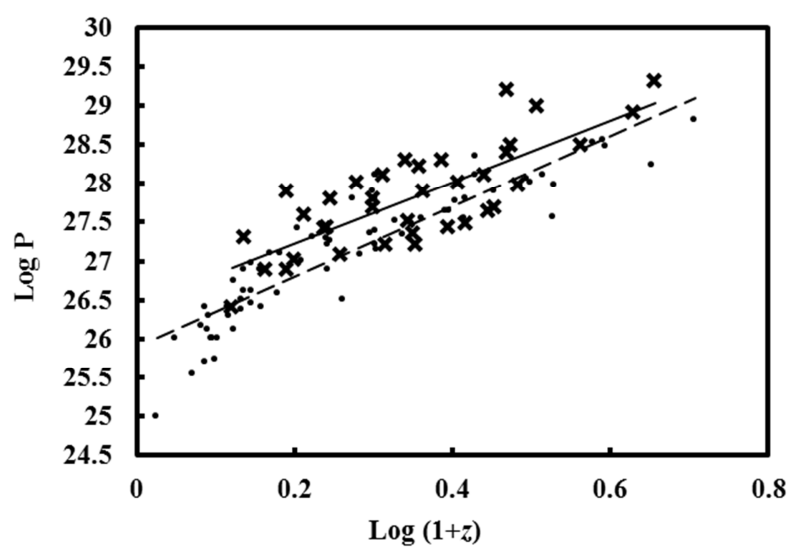

Figure 3. Scatter plot of the radio luminosity against the redshift (crosses \& solid line represent CSS quasars; while dots \& broken line represent CSS galaxies).

Moreover, we carry out linear regression of observed luminosities against observed redshifts of the CSS quasars and galaxies in our sample (Figure 3). Crosses and solid trend line represent CSS quasars, while dots and broken lines represent CSS galaxies. Results show relations of the form,

$$
\mathcal{P}_{b o l} \sim(1+z)^{\sigma}
$$

where $\sigma$ is 4 for the quasars and 5 for the galaxies. Correlation coefficients are respectively 0.8 and 0.9 .

This empirical relation shows that luminosity has excellent direct correlation with the redshift. However, this is different from the theoretical relation (equation (24)) which shows inverse relationship between the two parameters. This excellent direct correlation has been attributed to luminosity selection effects in which bright samples are found at high redshifts [13]. If actually the selection effect is removed/minimized we may be able to see the similarity of the two relations. But in this paper, we conclude that source radiated power may have some cosmological implications.

\section{Discussion and Conclusion}

We have used analytical methods in the previous sections with some plausible assumptions to find a relation (equation (7)) which plausibly may indicate a source size dependence on cosmological evolution. However, for the purpose of obtaining an empirical relation for comparison with the theory, we carry out linear regression analyses on the observed linear sizes against their respective observed redshifts for CSS quasars (Figure 1) and CSS galaxies (Figure 2). The power-law relations obtained are of the form, $\mathcal{D}_{0} \sim(1+z)^{\xi}$; where the index, $\xi$, is -2.5 and 2.9 for quasars and galaxies respectively. Correlation coefficients are generally marginal with 0.33 for quasars and 0.40 for galaxies.

In comparison with the theoretical relation (i.e. equation (7)), it can be seen that for the CSS quasars, the linear size $\left(\mathcal{D}_{0}\right)$ shows an inverse relationship with redshift $(z)$. This is similar to the theory. Hence, this similarity simply suggests that cosmological evolution may have some consequences on the source linear sizes.

However, the converse is found to be the case for the CSS galaxies. In this case the correlation is direct. One plausible explanation for this difference is that quasars are observed at higher redshifts than the galaxies'. Therefore, cosmological effects are expected to be more prominent on the quasars.

Furthermore, we used analytical methods again to obtain a theoretical relation (equation (24)) that shows relationship between luminosity and redshift. The relation shows that luminosity (or generally, radiated power) of a radio source falls off as $(1+z)^{-9}$. Hence, it shows that luminosity of a radio source may suggestively be modified by cosmological evolution.

Moreover, for the purpose of obtaining an empirical relation for comparison with the theory, we carry out linear regression analysis of observed luminosities against observed redshifts of the CSS quasars and galaxies in our sample (Figure 3). Results show relations of the form, $\mathcal{P}_{b o l} \sim(1+$ $z)^{\sigma}$; where $\sigma$ is 4 for the quasars and 5 for the galaxies. Correlation coefficients are respectively 0.8 and 0.9 .

This relation indicates that observed luminosity has excellent direct correlation with observed redshift. However, this is in contradiction to the obtained theoretical relation (i.e., equation (24)) which shows inverse relationship between the two parameters. This excellent direct correlation has been attributed to strong luminosity selection effects in which samples with high luminosities are found at high redshifts [13]. For larger radio sources, the effect was found to be $\approx 80 \%$ for quasars and $70 \%$ for galaxies [13]. So, CSS sources may not be an exception. Therefore, if the selection effects retaken care of, we may be able to see the comparability of the theoretical relation with the empirical relation. However, in the meantime, we conclude that source radiated power may have some cosmological implications just like we saw in the $\mathcal{D}_{0}-z$ relation.

\section{References}

[1] Mahatma, V. H., Hardcastle, M. J., Williams, W. L (2019) LoTSS DR1: Double-double Radio Galaxies in the HETDEX field. Astronomy and Astrophysics, 622, A13.

[2] Mingo, B. J., Croston, H., Hardcastle, M. J. (2019) Revisiting the Fanaroff-Riley Dichotomy and Radio Galaxy Morphology with the LOFAR Two-Meter Sky Survey (LoTSS). Monthly Notices of the Royal Astronomical Society, 488, 2701-2721.

[3] Hardcastle, M. J., Williams, W. L., Best, P. N. (2019) Radioloud AGN in the First LoTSS Data Release - The Lifetimes and Environmental impact of Jet-Driven Sources. Astronomy and Astrophysics, 622, A12. 
[4] Dabhade, P., Gaikwad, M., Bagchi, J. (2017) Discovery of Giant Radio Galaxies from NVSS: Radio and Infrared Properties. Monthly Notices of the Royal Astronomical Society, 469 (3), 2886-2906.

[5] Robson, I., Active Galactic Nuclei. (1996) Praxis Publishing Ltd, England.

[6] O'Dea, C. P. (1998) The Compact Steep Spectrum Sources and Gigahertz Peaked Spectrum Radio Sources. Publications of the Astronomical Society of the Pacific, 110, 493-532.

[7] Fanti, C., Fanti, R., Dallacasa, D. C., Schilizzi, R. T., Spencer, R. E., and Stanghellini, C. (1995) Are Compact Steep Spectrum Sources Young? Astronomy and Astrophysics, 302, $317-326$.

[8] Zhang, J., Zhang, H., Gan, Y., Yi, T., Wang, J. and Liang E. (2020) Jet Properties of Compact Steep Spectrum Sources and an Eddington-ratio-driven Unification Scheme of Jet Radiation in Active Galactic Nuclei. The Astrophysical Journal, 899, 2.

[9] Murgia, M. Fanti, R., Gregorini, L., Klein, U., Mark, K. H., and Vigotti, M. (1999) Synchrotron Spectra and Ages of
Compact Steep Spectrum Radio Sources,. New Astronomy Reviews, 345, 769-777.

[10] Cavalho, J. C. (1998) The Evolution of GHz-peaked Spectrum Radio Sources. Astronomy and Astrophysics, 329, 845-852.

[11] Jackson, C. A. (1999) Radio Source Evolution and Unified Schemes. Publications of Astronomical Society of Australia, $16,124-129$.

[12] Jeyakumar, S., Wiita, P. J., Saikia, D. J. and Hooda, J. S. (2005) Jet Propagation and the Asymmetries of CSS Radio Sources. Astronomy and Astrophysics, 432, 823-833.

[13] Ubachukwu, A. A. and Ogwo, J. N. (1999) Redshift and Luminosity Dependence of Linear Size of Compact Steep Spectrum Sources and the Quasar/galaxy Unification Scheme. Australian Journal of Physics, 52, 141-146.

[14] Ezeugo, J. C. and Ubachukwu, A. A. (2010) The Spectral Turnover - Linear Size Relation and the Dynamical Evolution of Compact Steep Spectrum Sources. Monthly Notices of the Royal Astronomical Society, 408, 2256-2260.

[15] Cavalho, J. C. 1998. "The Evolution of GHz-peaked Spectrum Radio Sources". Astronomy and Astrophysics, 329: 845-852. 